\title{
Air-to-Air Evaluation of an Amplified 802.11b Network
}

\author{
Robert A. Volesky, Brian A. Kish, Douglas O. Creviston, Jason W. Geitgey, Morikazu Kikuchi, Jason C. Vap \\ United States Air Force Test Pilot School \\ 220 South Wolfe Avenue \\ Edwards AFB, CA 93524-6485, USA \\ 661-277-9933 \\ robert.volesky@us.af.mil
}

Abstract - Students at the United States Air Force Test Pilot School $^{12}$. successfully completed a limited evaluation of an 802.11b air-to-air WiFi data link between two C-12C test aircraft. The test team determined the maximum range capability for 1 megabit per second (Mbps) and $11 \mathrm{Mbps}$ transmissions over the WiFi data link when transmitting at 1 or 5 Watts of amplifier power, corresponding to 0.32 and 1.58 Watts of effective isotropic radiated power, respectively. Diagnostic and performance statistic software on laptops were used to gather the data rate and performance statistics in flight, while post flight analysis was conducted to retrieve the data link range statistics. The flight test results closely matched the predicted maximum ranges found using an RF link prediction model, accounting for cabling losses, amplification, antenna gain, free space losses, amplifier receive gain, and the published SecNet $11^{\circledR}$ receiver sensitivity. Increased reception range could have been achieved using lower loss RF cabling and placing the amplifier closer the antenna. Of the available configurations tested (ad hoc, infrastructure and bridge), ad hoc was found the most reliable. The test team also demonstrated the capability of transmitting and receiving text files, still images, pre-recorded video, and streaming, live webcam video between the two test aircraft.

\section{TABLE OF ConTEnTs}

1. INTRODUCTION..................................................1

2. SYSTEM DESCRIPTION .........................................1

3. DATA LINK RECEPTION ENVELOPE .....................3

4. Data Link Performance Characteristics..6

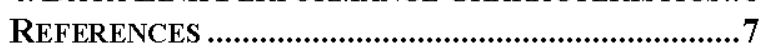

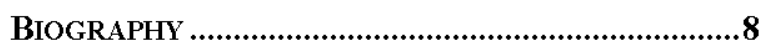

U.S. Government work not protected by U.S. copyright

IEEEAC paper \#1051, Version 1, 4 October 2007

\section{INTRODUCTION}

The ability to get real-time target imagery in the cockpit is invaluable to any pilot. The $802.11 \mathrm{~b}$ wireless data link is a potential low-cost commercial solution. To get operationally useful ranges, radio frequency antennas need to be amplified. The Federal Communications Commission limits transmission to 4 Watts effective isotropic radiated power. Taking all this into account, students at the United States Air Force Test Pilot School modified two C-12C aircraft to determine the reception envelope and performance characteristics in an air-to-air environment. Reference [1] is the full test report.

\section{SYSTEM DESCRIPTION}

The system consisted of a Haigh-Farr ${ }^{\circledR}$ aerial blade omnidirectional antenna [2] mounted on the top and bottom of the aircraft. Each antenna was connected to a 1 or 5 Watt (W) selectable Hyperlink Technologies ${ }^{\otimes}[3]$ amplifier located on the top of a rack in the C-12C cabin by RG-400 cabling. Figure 1 shows the amplifier was connected directly to Harris Corporation's SecNet $11^{\circledR}$ demonstration wireless network card [4] through RG-316 cabling and the wireless card was then inserted into the access point or laptop computer depending on the configuration being tested. Figure 2 shows the bottom antenna location, and Figure 3 shows the top antenna location. Dell Latitude ${ }^{\circledR}$ laptop PCs with Windows ${ }^{\circledR}$ XP, NetStumbler ${ }^{\circledR}$ software, Windows ${ }^{\circledR}$ XP Performance Monitor software, and a webbased camera for real-time video transfer using Microsoft ${ }^{\circledR}$ NetMeeting ${ }^{\otimes}$ completed the configuration being tested. Two GARMIN ${ }^{\circledR}$ GPS units were used for data link synchronization. One Itronix Duo-Touch ${ }^{\circledR}$ tablet PC in each aircraft monitored network performance and ambient noise in the $\mathrm{WiFi}$ spectrum. The airborne data link was transmitted at a frequency of $2.4 \mathrm{GHz}$ to $2.5 \mathrm{GHz}$. Either 1 or $5 \mathrm{~W}$ of amplification was used, which produced 0.32 or $1.58 \mathrm{~W}$ of effective isotropic radiated power. NetStumbler ${ }^{\circledR}$ software was used to gather signal-to-noise ratio (SNR) data. GPS position was displayed on a moving-map display using FalconView ${ }^{\mathbb{Q}}$. Microsoft ${ }^{\mathbb{Q}}$ NetMeeting $^{\mathbb{Q}}$ software was used to conduct text and video chat over the data link. Table 1 lists the components that were used during testing. 
Table 1: Components List

\begin{tabular}{|c|c|c|}
\hline Component & Model & Manufacturer \\
\hline $\begin{array}{l}\text { Aerial Blade } \\
\text { Antennas }\end{array}$ & $6030-2$ & Haigh-Farr $^{\circledR}$ \\
\hline $\begin{array}{l}\text { Selectable } 1 \text { or } 5 \\
\text { Watt Amplifiers }\end{array}$ & HA240105GAI-NF & $\begin{array}{l}\text { Hyperlink } \\
\text { Technologies }\end{array}$ \\
\hline GPS Receiver & GPSMAP 296 & Garmin $^{\circledR}$ \\
\hline Laptop & Latitude D620 & $\mathrm{Dell}^{\circledR}$ \\
\hline $\begin{array}{l}\text { Wireless } \\
\text { Network Card }\end{array}$ & $\begin{array}{l}\text { SecNet } 11^{\otimes} \text { Demo } \\
\text { Card }\end{array}$ & $\begin{array}{l}\text { Harris } \\
\text { Corporation }\end{array}$ \\
\hline $\begin{array}{l}\text { Duo Touch } \\
\text { Tablet PC }\end{array}$ & IX325 & Itronix $^{8}$ \\
\hline Web-Cameras & Quickcam $^{\circledR}$ & Logitech $^{\circledR}$ \\
\hline NetStumber $^{\circledR}$ & Version 0.4 .0 & Netstumbler.com \\
\hline $\begin{array}{l}\text { Windows }{ }^{\circledR} \text { XP } \\
\text { Perf Monitor }\end{array}$ & Version 5.1 & Microsoft $^{\circledR}$ \\
\hline NetMeeting ${ }^{\circledR}$ & Version 3.01 & Microsoft $^{\circledR}$ \\
\hline
\end{tabular}

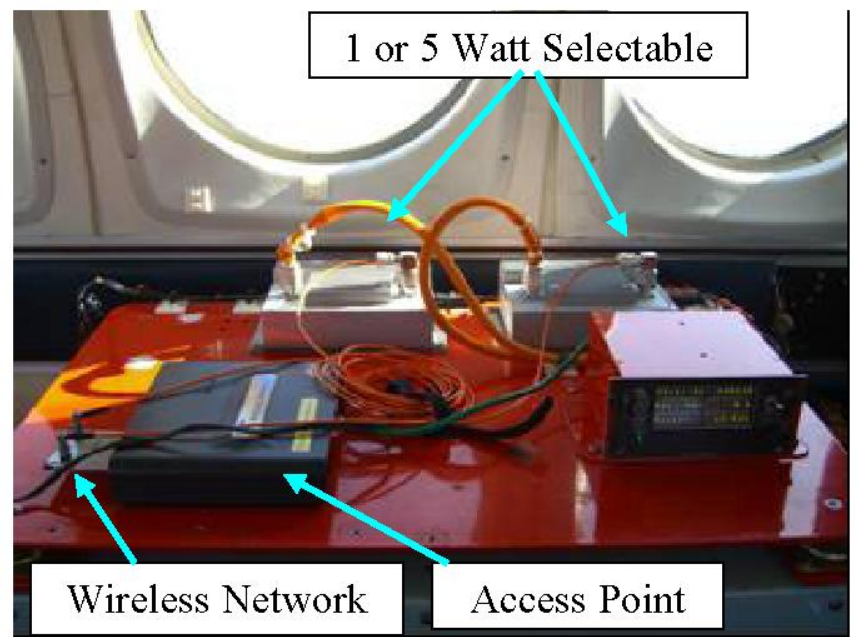

Figure 1 - Test Equipment Mounted to C-12C Rack

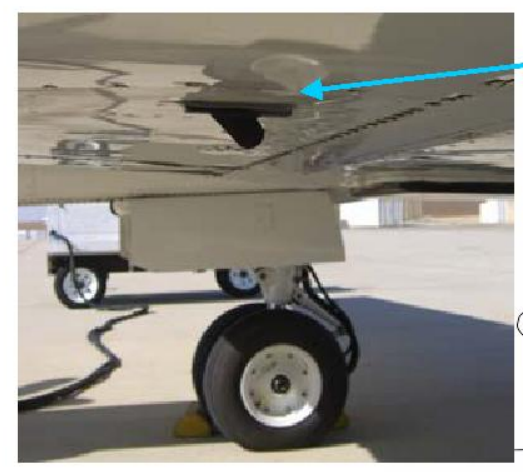

Photo of Installed Antenna

Figure 2 - Haigh-Farr ${ }^{\circledR}$ Antenna Position on Bottom of C-12C Test Aircraft

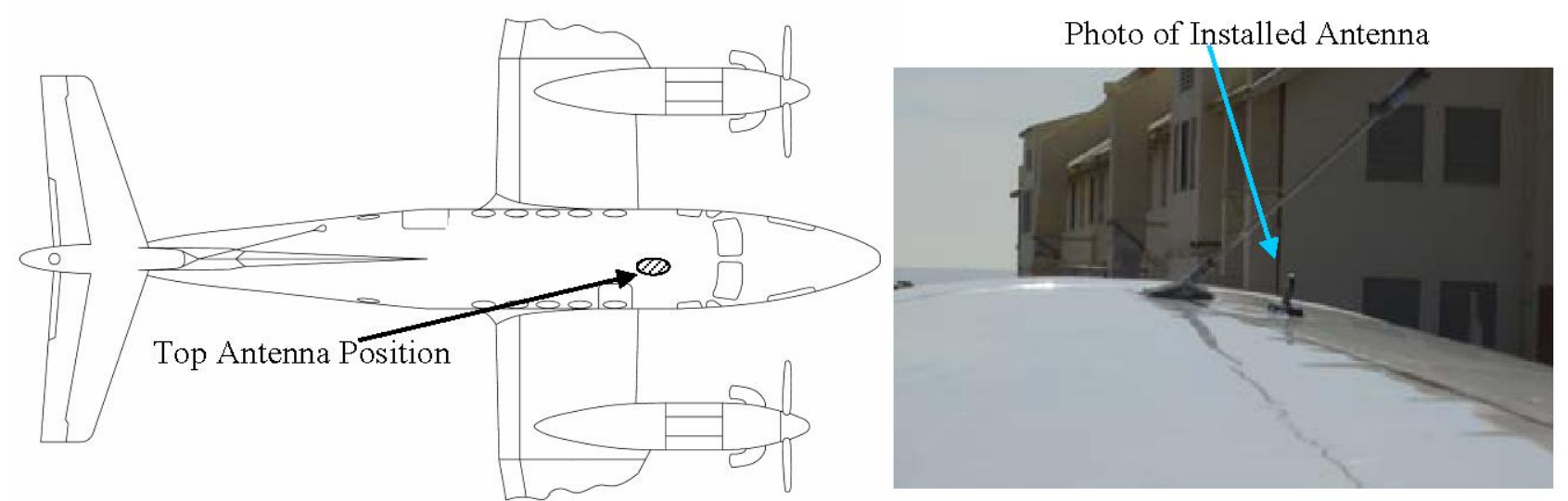

Figure 3 - Haigh-Farr ${ }^{\circledR}$ Antenna Position on Top of C-12C Test Aircraft 


\section{DATA LiNK RECEPTION ENVELOPE}

The first objective was to determine the air-to-air data link reception envelope between the two $\mathrm{C}-12 \mathrm{C}$ aircraft, while testing all three physical configurations of the data linkInfrastructure, Bridge, and Ad Hoc. Although each data link configuration was different, the flight test procedures in terms of pre-takeoff actions, flight test techniques (FTTs) used, and data collection techniques remained the same. To define the data link reception envelope, a set of FTTs were designed to both capture azimuth and elevation dependencies. Several user options of the data link were available, so the results attained are reported in an effectsbased fashion to clarify all the data link configuration considerations.

\section{Procedures}

Data link management duties were assigned to operators in each aircraft based upon whether it was the control or the host aircraft. The host aircraft was responsible for changing data rates, changing amplifications, maintaining the link, and monitoring the GPS data collection. The control aircraft was responsible for actively conducting data file transfers or signal-to-noise ratio (SNR) measurements, in addition to the responsibilities listed for the host aircraft. Due to the flight test workload requirements of the control aircraft, two flight test engineers (FTEs) were scheduled, when possible, to fly on that aircraft (test conductor and laptop operator). A single FTE was scheduled to fly on the host aircraft.

For all flight tests, the air-to-air data link was configured on the ground prior to takeoff. The following actions were completed before taxi: setting up the data link configuration; establishing over-the-air connectivity between $\mathrm{C}-12 \mathrm{~s}$; updating the laptop system clock with GPS time; configuring Windows ${ }^{\circledR}$ XP Performance Monitor to collect data rates and performance characteristics; starting NetStumbler ${ }^{\mathbb{B}}$; and setting altimeters to the local setting. Configuring the data link consisted of establishing physical connectivity to the amplifiers and setting the power level on the amplifiers (either 1 or $5 \mathrm{~W}$ ). Over-the-air connectivity was verified through conducting a file transfer. Each laptop was updated with GPS time to synchronize the aircraft GPS position files with the performance statistics log files. The Windows ${ }^{\circledR}$ XP Performance Monitor file was configured to collect all network interface characteristics - data transfer rates and performance statistics. The sample rate was set to 1 Hertz - the fastest rate available. NetStumbler ${ }^{\circledR}$ was configured from the control aircraft and was used to collect SNR data. The software could only be used during Infrastructure configuration testing due to architecture (physical configuration) requirements. The altimeters were set to local pressure to maintain a consistent test methodology.

Once all pre-flight actions were accomplished, an interval takeoff was conducted. A rejoin in extended trail was initiated following the takeoff. Data transfers were conducted throughout the climb to monitor the link performance and facilitate an efficient entry to the first test maneuver. All data transfers consisted of a data pull and a data push to maintain a steady, overall data transfer rate. All file transfers were initiated from the control aircraft $-\mathrm{a}$ data pull was a file transfer from the host aircraft to the control aircraft, while a data push was a file transfer from the control aircraft to the host aircraft. The overall data rate was higher when both a data push and a data pull were conducted at the same time. The full bandwidth available to the network was used ensured the data link was always data rate saturated.

The FTTs flown addressed one or more of the following areas: defining the reception envelope in 30 degree increments of azimuth; examining the effect of altitude separation; gaining statistical significance without increasing the number of the runs; examining antenna blanking events; and/or examining high noise effects. Visual depictions of the FTTs flown for this flight test are found in [1]. All outbound FTTs commenced with maximum available data rate and terminated 10 seconds after link break. All inbound FTTs started from a range greater than link break and required the laptop operator to initiate data transfers at the first indication of link availability.

\section{Configuration Effects}

All three physical data link configurations available for test were evaluated first during ground tests prior to flight test. Over 28 hours of ground tests suggested all three configurations would work under the conditions the data link would be exposed to during flight tests. However, only the Ad Hoc configuration was found robust (free of hardware/software resets) enough to continue flight testing. The Infrastructure configuration was the first physical configuration tested and was used to examine the best test method (data file transfers or SNR measurements) for determining the distance to link break - the point in which data file transfers could no longer be conducted. While the best test method was able to be isolated from the flight test dedicated to Infrastructure configuration testing, the configuration itself could not continue to be used due to the amount of time required to recover from the faults. The SecNet $11^{\circledast}$ card used in the laptop of the control aircraft had to be reset over five times in flight. Each time this occurred, the flight test maneuver had to be repeated because the data link was rendered totally inoperable during those events. 
The Bridge configuration was tested during the second flight test. Similar faults were encountered while testing in this configuration, and the same card reset procedures were executed to successfully recover the data link. The card requiring the resets was in the host aircraft (configured as the "slave").

The Ad Hoc configuration was tested during the third flight test. The same cards were used as during the Infrastructure and Bridge configurations. No faults were encountered during the third flight test or during any of the remaining four flight test sorties which performed Ad Hoc configuration testing. Due to the reliability of the Ad Hoc configuration, it was selected for the remainder of flight test efforts. During the first three flight tests, a relationship between the range reception and the selected data rate was discovered and is discussed in the data rate effects section.

\section{Data Rate Effects}

The first three flights demonstrated an increased data link reception range when a $1 \mathrm{Mbps}$ data rate was selected rather than AUTO data rate. The AUTO data rate mode was predicted to automatically reduce the data rate from 11 Mbps to $5.5 \mathrm{Mbps}$ to $2 \mathrm{Mbps}$ and then down to $1 \mathrm{Mbps}$ as SNR deteriorated with increasing range. While AUTO did reduce the data rate as range increased, the data link reception envelope was smaller than when $1 \mathrm{Mbps}$ was selected. Further testing was done to quantify the impact data rate selection had on the reception range. Two range values were determined for each maneuver flown. The full data rate range represents the range at which the data rate dropped below 90 percent of its highest value for at least five seconds. The other range measured was the link break range which is the range where the data link did not transfer any data for at least five seconds. Figure 4 illustrates the 95 percent confidence interval (CI) for link break ranges achieved with $1 \mathrm{Mbps}, 11 \mathrm{Mbps}$ and AUTO data rate selections. There was not a significant difference between the AUTO and $11 \mathrm{Mbps}$; however, the range reception nearly doubled when $1 \mathrm{Mbps}$ was selected.

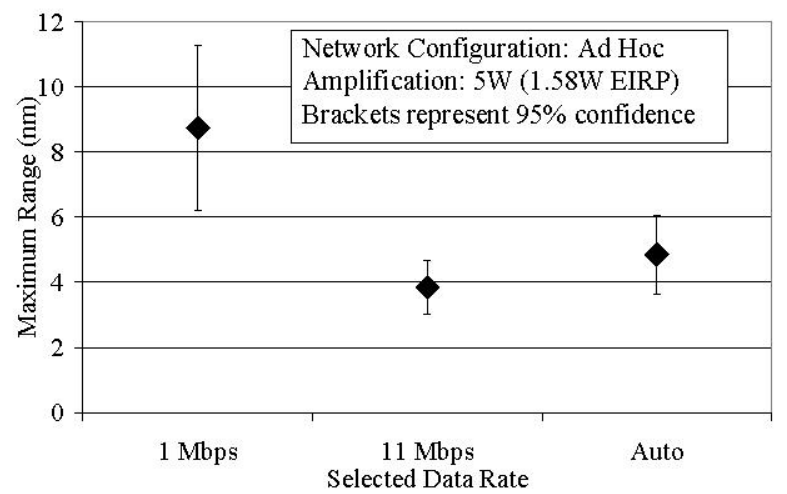

Figure 4 - Reception Range Dependence on Data Rate

\section{Actual Data Rates}

While the data rates selected were AUTO, $11 \mathrm{Mbps}, 5.5$ Mbps, $2 \mathrm{Mbps}$, and $1 \mathrm{Mbps}$, the actual over-the-air data rates were much lower. The actual data rates are identified in Table 2. The data rates were a function of the physical configuration (Infrastructure, Bridge, or Ad Hoc). Since both Infrastructure and Bridge configurations used an access point, the data rates were the same and limited by that device. Knowing the over-the-air data rate gives the user baseline information for developing future applications.

Table 2. Actual Data Rates

\begin{tabular}{|c|c|c|c|}
\hline \multirow{2}{*}{$\begin{array}{c}\text { Selected } \\
\text { Data Rate }\end{array}$} & \multicolumn{3}{c|}{ Actual Data Rates (Mbps) } \\
\cline { 2 - 4 } & Infrastructure & Bridge & Ad Hoc \\
\hline AUTO & 3.3 & N/A & 4.4 \\
\hline $11 \mathrm{Mbps}$ & 3.3 & 3.3 & 4.4 \\
\hline $5.5 \mathrm{Mbps}$ & 3.0 & 3.0 & 3.0 \\
\hline $2 \mathrm{Mbps}$ & 1.4 & 1.4 & 1.4 \\
\hline $1 \mathrm{Mbps}$ & 0.7 & 0.7 & 0.7 \\
\hline
\end{tabular}

\section{Power Effects}

Amplification was selectable between $1 \mathrm{~W}$ and $5 \mathrm{~W}$. Selectable amplifiers were used for the flight test, so the effects of high noise environments outside the Edwards Restricted Airspace could be observed. Transmit power for all $802.11 \mathrm{~b}$ devices were limited by Federal Communications Commission (FCC) part 15.247 to $1 \mathrm{~W}$ or $30 \mathrm{dBm}$ of transmitter power delivered to a $6 \mathrm{~dB}$ isotropic antenna, effectively $36 \mathrm{dBm}$ of effective isotropic radiated power (Ref 1). Clearance to exceed the FCC criteria could be granted inside the Edwards Restricted Airspace by the Edwards AFB Spectrum Management Office; however, their jurisdiction was limited to Edwards Restricted Airspace. For this reason, $1 \mathrm{~W}$ amplification was used outside the restricted airspace for high noise testing and 5 W amplification was used inside the restricted airspace to quantify the effects amplification had on the reception range. Some additional $1 \mathrm{~W}$ testing was conducted in the Edwards Restricted Airspace to provide a baseline reception range for comparison with the high noise test points.

\section{Measured Range}

The factors available for testing were data link configuration, data rate, and power. The decision to test at $11 \mathrm{Mbps}$ and $1 \mathrm{Mbps}$ was made to examine the extremes of the data link's utility. The $11 \mathrm{Mbps}$ data rate offered the greatest available data link utility in terms of throughput available for applications, while the $1 \mathrm{Mbps}$ data rate 
offered the longest range available to the data link. The decision to conduct $1 \mathrm{~W}$ and $5 \mathrm{~W}$ testing was based on examining the impact of transmit power and high levels of $\mathrm{RF}$ noise on the data link reception range. Given those decisions, the Ad Hoc configuration was placed into the following testable configurations: $11 \mathrm{Mbps} / 5 \mathrm{~W}, 1 \mathrm{Mbps} / 5$ $\mathrm{W}, 11 \mathrm{Mbps} / 1 \mathrm{~W}$, and $1 \mathrm{Mbps} / 1 \mathrm{~W}$.

An analysis was conducted on the four available configurations to develop an orthogonal test matrix of varying range and varying elevation maneuvers. The analysis was focused on examining the azimuth and elevation dependence of the data link reception envelope. A single run of each test point was planned; however, some maneuvers were conducted multiple times due to airspace availability and air traffic conflicts during maneuvers. Overall, the results of the entire set of maneuvers were statistically analyzed to determine the data link reception envelope and its dependence on azimuth and elevation.

A total of 28 test points were evaluated for the $5 \mathrm{~W}$ amplification test points. From the analysis, no azimuth or elevation dependencies yielded a statistical significance. Although the raw test results appeared to have an azimuth dependence, particularly during tail-to-tail maneuvers, there were not enough test runs to yield a statistical significance of this phenomenon. Free of azimuth and elevation dependencies, the range results from each maneuver were combined and analyzed as an amalgam to determine the 95 percent $\mathrm{CI}$ of the reception envelope for the $11 \mathrm{Mbps}$ and 1 Mbps data rates. The 95 percent $\mathrm{CI}$ for $11 \mathrm{Mbps}$ and 1 Mbps data rates under $1 \mathrm{~W}$ and $5 \mathrm{~W}$ amplification can be seen in Figure 5. The $11 \mathrm{Mbps}$ data rate region to link break of the reception envelope was between 3.0 to $4.7 \mathrm{~nm}$ between the aircraft, and the $1 \mathrm{Mbps}$ data rate region to link break was between 6.2 to $11.3 \mathrm{~nm}$. In general, the large intervals were primarily due to the instances in which the tail-to-tail test points yielded shorter ranges, while many of the 60 degree varying range and varying elevation test points yielded longer ranges. Flying more replicates could reduce the CIs and provide a realistic azimuth and elevation dependence of the data link reception envelope. These dependencies are necessary to reveal the installed antenna effects, but more importantly they provide the operator with the best and worst locations of the reception envelope.

The same analysis was conducted on the 18 flight test maneuvers used to collect and define the data link reception envelope under $1 \mathrm{~W}$ of amplification. No azimuth or elevation dependencies were present in the analysis. The 95 percent Cls for $1 \mathrm{Mbps}$ and $11 \mathrm{Mbps}$ data rates can be seen in Figure 5. The $11 \mathrm{Mbps}$ data rate region to link break of the data link reception envelope was between 0.76 to 2.3 $\mathrm{nm}$ between the aircraft and, the $1 \mathrm{Mbps}$ data rate region to link break was between 2.4 to $4.6 \mathrm{~nm}$.

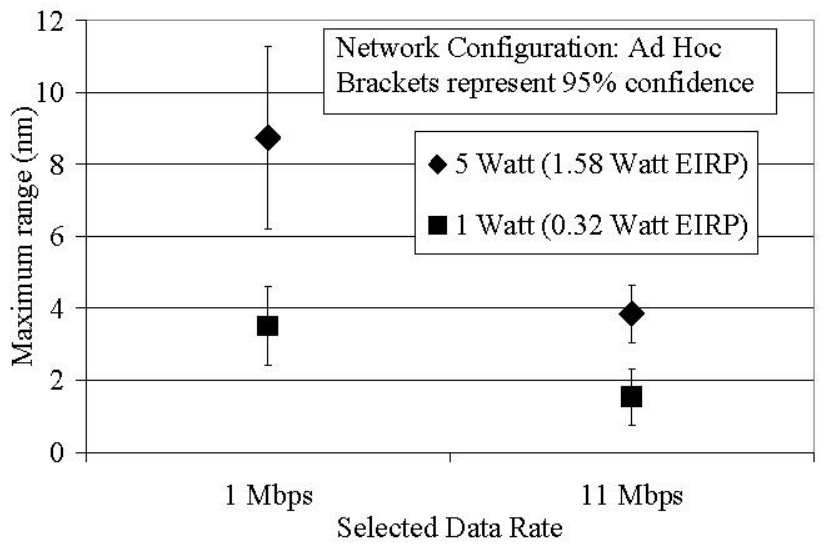

Figure 5 - Data Link Reception Range

\section{Losses and Noise Effects}

One of the most important attributes of an operational tactical data link is range. Good range performance relaxes the operational bounds a pilot must regularly consider to maintain connectivity. The standard $\mathrm{RF}$ range equation was applicable to this test. In this equation, range was a function of amplification, antenna gain, cabling losses, and free-space losses. Based on test team operational experience, the maximum ranges for maximum data rate and for basic link maintenance were smaller than that desired for an operational data link. This was because of the system's low effective radiated power, stemming from low amplification levels and high cable losses. The amplification levels were limited by FCC regulations concerning $802.11 \mathrm{~b}$ applications. The tests were conducted with the maximum amplification levels allowed under those regulations. Cabling losses were a function of cable type and cable length. Cabling alone accounted for a $20-\mathrm{dB}$ loss.

Amplified output power was fixed at either $1 \mathrm{~W}$ or $5 \mathrm{~W}$ (selectable). Approximately $16 \mathrm{~dB}$ of cabling losses were present between the transmitting amplifier and the receiving amplifier. After the fixed receiving amplifier gain, a further $4 \mathrm{~dB}$ of loss was present before connection to the computer. In total, this led to $20 \mathrm{~dB}$ of loss between the transmitting amplifier and the receiving computer. This signal reduction reduced the data link range from what could have been possible for this test. For future applications, cabling losses of this magnitude will greatly reduce range and decrease operational utility. An additional technique to minimize cable losses would be to put the amplifiers as near the antennas as possible.

The achievable data rate was a function of SNR. The previous discussion was concerned with the power of the signal. The noise level will also be important to future applications, but will be difficult to predict and even harder to control. Testing showed high sensitivity to noise, especially when operating near the maximum range. The 
test team did not predict significant effects due to noise when operating at the test altitude of 20-25,000 feet mean sea level (MSL) because of the low power of home WiFi networks. In actuality, noise appeared to be a factor even at those altitudes due to other devices transmitting $\mathrm{RF}$ energy in the $2.4-2.5 \mathrm{GHz} 802.11 \mathrm{~b}$ frequency band. While those devices could not be identified, the Itronix Duo-Touch Tablet PCs carried in the test aircraft incorporated spectrum analysis tools which identified varying noise levels at altitude. Unfortunately, the spectrum analysis recording and playback functionality was limited, so noise level was gathered by the FTE in the aircraft only by visually watching the noise level and annotating when more than -60 $\mathrm{dBm}$ of noise was encountered. As a specific example, higher noise was observed in the vicinity of China Lake/Ridgecrest on flight \#5 which caused the data link to drop while within approximately $3 \mathrm{~nm}$ lateral spacing of the developed area. Outside of that area of noise, the data link quality was good and high data rate was achieved.

To further quantify the effects of noise, several runs were made at low altitude (3,000-4,000 feet above ground level) over the Lancaster/Palmdale urban area. These test points were conducted in the Ad Hoc configuration with $1 \mathrm{~W}$ amplification and $1 \mathrm{Mbps}$ and $11 \mathrm{Mbps}$ data rate selected. Using the constant relative position FTT, the maximum range for data link operation was 2,000 feet for $1 \mathrm{Mbps}$ and 1,000 feet for $11 \mathrm{Mbps}$. On the same day, with the same configuration, similar test points were performed at an altitude of 20,000 feet MSL in the relatively uninhabited areas of the Edwards AFB controlled airspace. The maximum range for data link operation was $3.5 \mathrm{~nm}(21,000$ feet) for $1 \mathrm{Mbps}$ and $0.7 \mathrm{~nm}$ (4,200 feet) for $11 \mathrm{Mbps}$. This shows an order-of-magnitude difference between achieved data link range in high and low noise environments, substantiating the expectation that noise level would have a large effect on data link performance. Failure to predict and plan for expected maximum noise levels may result in inadequate link performance in high-noise environments.

\section{Data Link Performance Characteristics}

The performance characteristics were assessed quantitatively and qualitatively. Data link performance statistics were collected during all the test points directed at determining the air-to-air data link reception envelope. Additionally, specific test points were flown to gather data on performance related tasks. The results of this testing revealed the efficiency and utility of the data link.

\section{Procedures}

The laptops on the host and control aircraft were both configured to collect performance statistics using the Windows ${ }^{\circledR}$ XP Performance Monitor software. The parameters collected were from the network interface statistics: output queue length; packets outbound discarded, packets outbound errors, and packets received errors. These primary statistics describe how efficiently the data link was performing during data transfer operations, using transmission control protocol (TCP) or unit datagram protocol (UDP). Errors and discarded packets demonstrate reduced efficiency and the output queue length will increase as error rates build. The difference between the two protocols is application based - TCP is used when errors in raw data after packaged and received is not tolerable, and UDP is used when errors in the raw data after packaged and received is tolerable. TCP statistics were collected during still image, text file, and pre-recorded video file transfers. UDP statistics were collected during webcam operations live, streaming video transfers. UDP reduces the data packaging requirements of outbound data because of the presence of human interpretation in the application. The human factor allows for interpretation that is not present in strict data transmissions. For instance the human ear can understand a mispronounced word based on the context the word was used in. The same can be said about video quality. The human eye can look past missing pixels and still glean the overall intended picture.

Another quantitative measure of performance was the time required to transfer operationally representative file types. Actual time measurements were taken during a still image, text file, and pre-recorded video file transfer within the applicable region (high data rate or low data rate) of the data link reception envelope.

The qualitative performance of the data link was assessed using three live data transfers. Live video was streamed using webcams. Text chat was performed throughout the testing using Microsoft ${ }^{\circledR}$ NetMeeting ${ }^{\circledR}$. GPS position of each aircraft was transmitted over the network to the other aircraft and displayed in real-time on FalconView ${ }^{\circledR}$.

\section{Data Link Performance Results}

Overall, the network interface statistics reflected high network efficiency for all data rates and ranges. The output queue length, packet outbound errors, discarded packets received, packet errors received, UDP errors, and transmission control protocol (TCP) errors consistently remained at zero, demonstrating an efficient use of the available data rate during all transmissions. All qualitative tasks were performed effectively (free of software resets) at all data rates and ranges where the network existed. The statistics of performance file transfers made in the $1 \mathrm{Mbps}$ and $11 \mathrm{Mbps}$ reception ranges are listed in Table 3. 
Table 3. Performance Testing - Time to Transfer

\begin{tabular}{|c|c|c|c|c|}
\hline $\begin{array}{c}\text { File } \\
\text { Type }\end{array}$ & $\begin{array}{c}\text { File Size } \\
(\mathrm{kB})\end{array}$ & $\begin{array}{c}\text { Selected } \\
\text { Data Rate } \\
(\mathrm{Mbps})\end{array}$ & $\begin{array}{c}\text { Range } \\
(\mathrm{nm})\end{array}$ & $\begin{array}{c}\text { Time to } \\
\text { Transfer } \\
(\mathrm{sec})\end{array}$ \\
\hline $\begin{array}{c}\text { Text } \\
\text { File }\end{array}$ & 2.1 & 1 & 5.2 & 3 \\
\hline $\begin{array}{c}\text { Still } \\
\text { Image }\end{array}$ & 120 & 1 & 5.2 & 33 \\
\hline $\begin{array}{c}\text { Small } \\
\text { Video }\end{array}$ & 1280 & 1 & 5.2 & 85 \\
\hline $\begin{array}{c}\text { Large } \\
\text { Video }\end{array}$ & 18000 & 11 & 2.3 & 32 \\
\hline
\end{tabular}

\section{Conclusion}

The test team performed seven formation test flights encompassing 35 flight hours during March 2007 to determine the reception envelope and the performance of an airborne $802.11 \mathrm{~b}$ Wireless Fidelity (WiFi) data link as a function of range, elevation, azimuth, data rate, amplification level, link configuration, and noise level. The data link envelope was defined in terms of azimuth angle, elevation angle, and slant range. The performance of the data link was defined in terms of data rate, TCP and UDP error statistics, and network health statistics.

The 95 percent confidence interval for range with $11 \mathrm{Mbps}$ and $5 \mathrm{~W}$ of amplification (1.58 W EIRP) selected was 3.0 to 4.7 nautical miles with a maximum actual data rate of 4. $4 \mathrm{Mbps}$. The 95 percent confidence interval for range with $1 \mathrm{Mbps}$ and $5 \mathrm{~W}$ (0.32 W EIRP) selected was 6.2 to 11.3 $\mathrm{nm}$ with a maximum actual data rate of $0.7 \mathrm{Mbps}$. Performance characteristics of the data link were measured while sending text files, chat data, still images, pre-recorded video, and streaming webcam video between the two aircraft. Network health statistics were gathered for both TCP and UDP activities. Overall, the network health statistics reflected high network efficiency for all data rates and ranges.

Testing was performed on a WiFi network configured in three different ways: Ad Hoc, Infrastructure, and Bridge. The Ad Hoc configuration was found to be the most reliable and capable. Hardware errors associated with the SecNet1 $1^{\otimes}$ WiFi cards were encountered in the Infrastructure and Bridge modes which were frequent, persistent, and highly disruptive to the test. The effect of background noise level on data link performance was investigated by comparing maximum data link range in high and low noise levels. As expected, the maximum data link range in the high noise environment was an order-ofmagnitude less than that obtained in the low noise environment. An unexpected effect was the variation in noise level and corresponding data link maximum range in the low noise environment.

The data rate selected was found to have an impact on the reception range achieved. There were small differences between the range results of the automatic (AUTO) and 11 Mbps selected data rates. However, $1 \mathrm{Mbps}$ yielded twice the reception range of AUTO. The actual data rates did not match the selected data rates available to each physical configuration. The selections available were: AUTO, 1 Mbps, $2 \mathrm{Mbps}$, $5.5 \mathrm{Mbps}$, and $11 \mathrm{Mbps}$. The observed data rates were much lower than the selected data rates. Knowing the over-the-air data rate gives the user baseline information for developing potential applications for use across the data link.

\section{REFERENCES}

[1] Limited Evaluation of an $802.11 \mathrm{~b}$ Air-to-air Wireless Datalink, Project "Have HALO II" Technical Report, AFFTC-TIM-07-01, USAF Test Pilot School, Edwards AFB, June 2007

[2] Haigh-Farr Blade Antenna Data Sheet, Haigh-Farr Inc., Bedford, NH, 31 Jan 2007. http://www.haighfarr.com/datasheets/blade.pdf.

[3] $2.4 \mathrm{GHz}$ High Power WiFI Amplifiers with Switchable Power Control, Hyperlink Technologies, 7 Jun 2007. http://www.hyperlinktech.com/web/24ghz.

[4] SecNet1 $1{ }^{\circledR}$ Plus Secure Wireless LAN PC Card, Harris Corp., Melbourne, FL, 7 Feb 2007. http://www.govcomm.harris.com/securecomm/products/pc-card.html. 


\section{BIOGRAPHY}

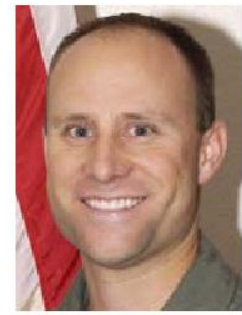

Capt Robert Volesky is a Flight Test Engineer at the 411th Flight Test Squadron at Edwards AFB, CA. His research interest includes wireless communications. Capt Volesky obtained a B.S. degree in Electrical Engineering from the United States Air Force Academy in 2000, and an M.S. in Electrical Engineering from Iowa State in 2001. He is a member of Tau Beta Pi, the Association of Old Crows, and the Society of Flight Test Engineers

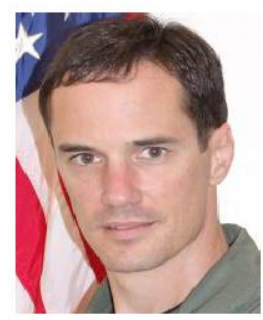

Lt Col Brian Kish is an instructor at the Air Force Test Pilot School. His research interests include control theory and operations research. He is the author of one book chapter and 12 articles in the areas indicated above. Lt Col Kish has a B.S. degree in Aerospace Engineering from Illinois Institute of Technology, and he has an M.S. and Ph.D. from the Air Force Institute of Technology. He is a member of AIAA and the Society of Flight Test Engineers.

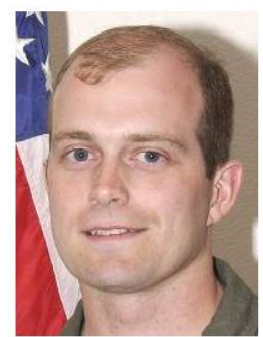

Maj Douglas Crevistion is a Test Pilot at the 416th Flight Test Squadron at Edwards AFB, CA. His research interests include gas turbine engines and data links. Maj Creviston has a Bachelor of Aeronautical Engineering from the United States Air Force Academy in 1997, and an M.S. in Aeronautical and Astronautical Engineering from the Massachusetts Institute of Technology in 1999. He is a member of the Society of Experimental Test Pilots.

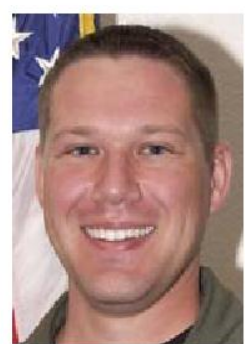

Capt Jason Geitgey is a Flight Test Engineer at the 46th Test Squadron at Eglin AFB, FL. His research interest includes control theory and sensor and defensive aircraft systems. Capt Geitgey obtained a B.S. degree in Aerospace Engineering from the University of Cincinnati in 1998, and an M.S. in Aeronautical Engineering from the Air Force Institute of Technology in 2006. He is a member of AIAA and the Society of Flight Test Engineers.

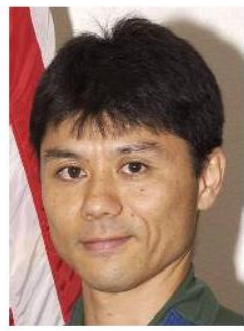

Maj Morikazu Kikuchi is a Test Pilot at the flight test squadron at Gifu $\mathrm{AB}$ in Japan. His research interests include upgrading F-15J's avionics. Maj Kikuchi obtained a B.S. degree in mechanical engineering from Aoyama Gakuin University in 1994. He is a member of the Society of Experimental Test Pilots.

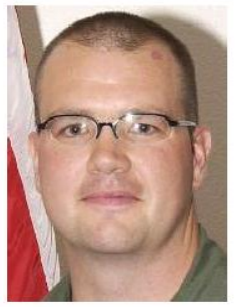

Capt Jason Vap is a Flight Test Engineer at the 417th Flight Test Squadron at Edwards AFB, CA. His research interests include semiconductor materials and devices. Capt Vap obtained B.S. and M.S. degrees in Electrical Engineering from the University of Wyoming in 2002 and 2004, respectively. He is a member of the Society of Flight Test Engineers. 